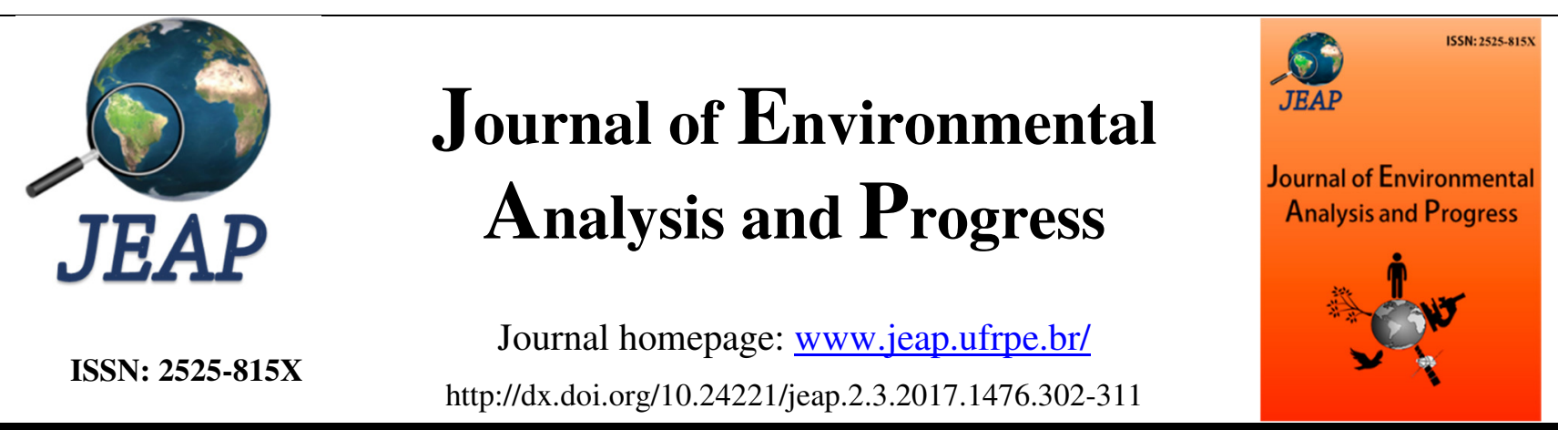

\title{
Definição de ambientes de produção para a cultura da Atriplex no estado de Pernambuco
}

\section{Definition of production environments for the culture of Atriplex in the State of Pernambuco}

\author{
Carlos Tiago Amâncio Rodrigues ${ }^{\mathrm{a}}$, Thieres George Freire da Silva ${ }^{\mathrm{b}}$, Herica Fernanda de Sousa \\ Carvalho $^{\mathrm{c}}$, Magna Soelma Beserra de Moura ${ }^{\mathrm{c}}$
}

a Universidade Federal do Vale do São Francisco-UNIVASF, Av. José de Sá Maniçoba, s/n, Centro, Petrolina-PE. CEP: 56304-917. E-mail: tiagoamancio@ hotmail.com.

${ }^{\text {b } U n i v e r s i d a d e ~ F e d e r a l ~ R u r a l ~ d e ~ P e r n a m b u c o-U F R P E, ~ U n i d a d e ~ A c a d e ̂ m i c a ~ d e ~ S e r r a ~ T a l h a d a-U A S T, ~ A v e n i d a ~ G r e g o ́ r i o ~}$ Ferraz Nogueira, S/N, José Tomé de Souza Ramos, Serra Talhada-PE. CEP: 56909-535. Emailthieresfreire@yahoo.br.

c Embrapa Semiárido, Rodovia BR-428, Km 152, s/n, Zona Rural, Petrolina-PE. CEP: $56302-970$. hericafernanda_17@hotmail.com; magna.moura@embrapa.br.

\begin{abstract}
A R T I C L E I N F O
Recebido 04 Jul 2017

Aceito 28 Jul 2017

Publicado 31 Jul 2017

A B S T R A C T

Identifying environments conducive to the exploration of crops is essential to the detriment of the strategic planning of the state, aiding in the formation of public policies and decision making. In this context, one of the species of great importance in arid and semi - arid regions is the Atriplex nummularia, which has potential forage, presenting an excellent tolerance to water stress and adverse conditions of salinities. In view of the context, this study had the objective of identifying areas with productive potential for the cultivation of "erva sal" (A. nummularia) in the state of Pernambuco. For that, the pedoclimatic zoning was elaborated, based on the climatic requirements of the crop and mapping of soils of the State. Monthly climatological data of rainfall and temperature observed and estimated as a function of those of the planialtimetric coordinates of 291 pluviometric stations were used. The maps of pedoclimatic zoning and productivity were elaborated using the Geographic Information System (GIS). The identification of the pedoclimatically suitable areas for cultivation, and the estimation of productivity at distinct cut and density intervals, were mapped. From the results it was found that, conditions of air temperature above $24^{\circ} \mathrm{C}$, and precipitation below $1200 \mathrm{~mm}$ are ideal for the cultivation of salt grass, with some areas restricted to the soil. Although some regions presented low productivity, most of the State of Pernambuco presented aptitude for the cultivation of "erva sal".
\end{abstract}

Keywords: Agroclimatic zoning, Atriplex nummularia, semi-arid.

\footnotetext{
R E S U M O

Identificar ambientes propícios para exploração de culturas é essencial em detrimento do planejamento estratégico do estado, auxiliando na formação de politicas públicas e tomada de decisão. Nesse contexto, uma das espécies de grande importância em regiões áridas e semiáridas é a Atriplex nummularia, que possui potencial forrageiro, apresentando ótima tolerância ao estresse hídrico e condições adversas de salinidades. Diante do contexto, este estudo teve como objetivo realizar a identificação de áreas com potencial produtivo para o cultivo da "erva sal" ( $A$. nummularia) no estado de Pernambuco. Para tanto, foi elaborado o zoneamento
} 
pedoclimático, a partir das exigências climáticas da cultura e mapeamento de solos do Estado. Foram usados dados climatológicos mensais de precipitação pluvial e temperatura observada e estimada em função das das coordenadas planialtimétricas, de 291 postos pluviométricos. Os mapas do zoneamento pedoclimático e produtividade foram elaborados usando o Sistema de Informações Geográficas (SIGs). A identificação das áreas pedoclimaticamente aptas ao cultivo, e a estimativa da produtividade em intervalos de corte e densidade distintos, foram mapeados. A partir dos resultados constatou-se que, as condições de temperatura do ar acima de $24^{\circ} \mathrm{C}$, e precipitação abaixo de $1200 \mathrm{~mm}$ são ideais para o cultivo da erva sal, com algumas áreas restrita em relação ao solo. Embora algumas regiões apresentaram baixa produtividade, a maior parte do estado de Pernambuco apresentou aptidão ao cultivo da "erva sal".

Palavras-Chave: Zoneamento agroclimático, salinidade, semiárido.

\section{Introdução}

A espécie Atriplex nummularia, conhecida como erva sal, planta halófita, originária da Austrália, pertence à família Chenopodiaceae, é uma das mais de 400 espécies do seu gênero distribuída em diversas regiões árida e semiárida do mundo (Leal, 2005). Destaca-se por apresentar fácil estabelecimento, elevado potencial forrageiro, boa aceitabilidade e suporte proteico aos animais, especialmente os ruminantes, ovinos e caprinos (Aganga et al., 2003; Leal et al., 2008). Pode ser utilizada no paisagismo e na floricultura como planta ornamental (Maia et al., 2003; Silva et al., 2003).

A implementação desta espécie em áreas ameaçadas pela degradação e desertificação, na forma de plantios isolados para fitoremediação de solos salinos-sódicos, tornando uma alternativa de recuperação é uma vantagem marcante desta cultura. Possui baixo custo e, sobretudo, não agride o meio ambiente, quando comparado ao uso de corretivos químicos (Leal et al., 2008). A mesma possui potencial para a produção abundante de biomassa, acumulando grande quantidade de sais na parte aérea, em seus tecidos, possibilitando vários cortes, e ainda é tolerante a um elevado déficit hídrico (Souza, 2010).

Estudos realizados sub condições de região semiárida por Porto \& Araújo (1999) constataram rendimento forrageiro de 6,5 t MS/ha, sendo 69,5, 15,0 e 15,5\% de folhas, caules finos e grossos, respectivamente. A composição química desse material foi de 30,7\% de MS, 14,9\% de PB, $56,7 \%$ de DIVMS, 50,2\% FDN e $19,45 \%$ de matéria mineral (cinzas). De acordo com estudos realizados pelo Instituto Agronômico de Pernambuco, a A. nummularia pode ser usada como recurso forrageiro importante na complementação da dieta de ruminantes, graças ao seu valor nutritivo em torno de $17 \%$ de proteína, e elevada digestibilidade, por volta $70 \%$. Além disso, apresenta taxas de absorção de sais solúveis que atingem 1,15 t.ha $^{-1}$. ano $^{-1}$ (Porto et al., 2001).

Portanto, a produção da erva sal constitui uma atividade de grande interesse para os produtores agropecuários. Porém, em Pernambuco, esta cultura ainda não tem sido explorada com todo o potencial de que se dispõe o estado, em relação às condições de solo e clima. Assim, o estudo de zoneamento agrícola, ganha valor, sobretudo, que é uma ferramenta de extrema importância, identificando áreas mais propícias a implantação da cultura.

Nesse contexto, visando minimizar os riscos relacionados aos fenômenos climáticos permitindo melhorar a tomada de decisão no momento da implantação de culturas, torna-se fundamental o direcionamento de pesquisas, sobretudo no que se refere à identificação de ambientes propícios para produção, gerando informações para a expansão de suas áreas de cultivo. No entanto, este estudo teve como objetivo realizar a identificação de áreas com potencial produtivo para o cultivo da Erva Sal (A. numularia) no estado de Pernambuco.

\section{Material e Métodos}

O estudo foi realizado para o estado de Pernambuco, localizado entre as coordenadas: Latitudes $7^{\circ} 20^{\prime}$ a $9^{\circ} 29^{\prime} \mathrm{S}$ e Longitudes de $34^{\circ}$ $30^{\prime}$ a $41^{\circ} 21^{\prime} \mathrm{W}$, limitado pelos estados da Paraíba (N), Ceará (NO), Alagoas (SE), Bahia (S) e Piauí (O), banhado pelo oceano Atlântico (L). O estado está subdividido em cinco mesorregiões: Metropolitana do Recife (5), Zona da Mata (4), Agreste (3), Sertão (1) e São Francisco (2), como podem ser visto na Figura 1. O estado tem dois tipos de climas, segundo a classificação de Koppen, As' e BSh. O clima As' é tropical, com chuvas de outono-inverno, domina a porção oriental do estado. E o clima BSh, caracteriza-se como semiárido, quente, dominando as porções central e ocidental do estado. 

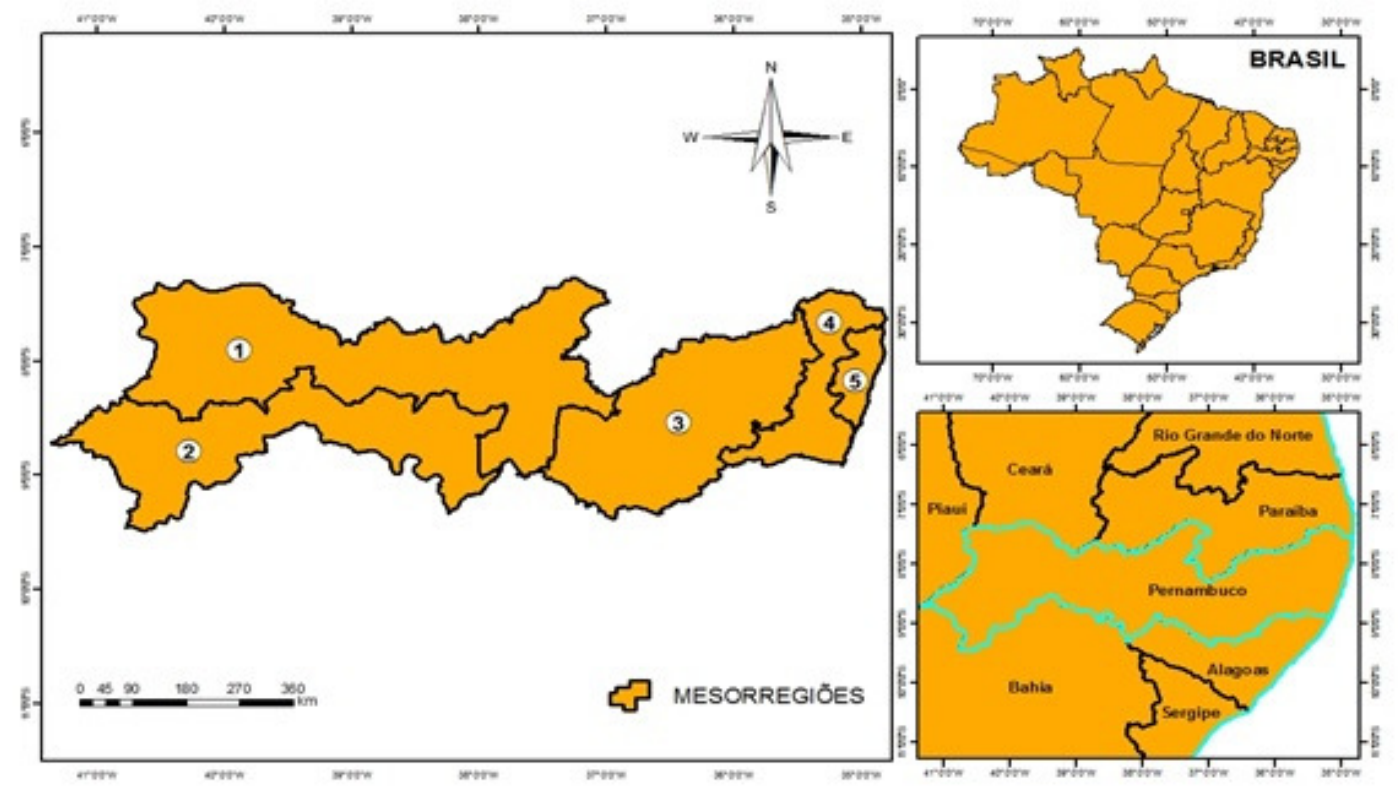

Figura 1. Localização da área de estudo no estado de Pernambuco, Brasil. Fonte: elaborado pelo o autor. (2017).

Para a realização do estudo foi organizado um banco de dados climatológicos de 291 postos de observação. O período de dados corresponde aos anos de 1961 a 1990. A série de dados climática normal (> 30 anos) de precipitação pluviométrica foi adquirida do Departamento de Ciências Atmosférica/Universidade Federal de Campina Grande-DCA/UFCG. Por sua vez, para os locais que não se dispunham da temperatura, a mesma foi estimada usando as equações desenvolvidas por Alvares et al. (2012), em função dos valores de longitude, latitude e altitude (m).

Os dados de solos foram obtidos a partir do banco de dados do Zoneamento Agroecológico do Estado de Pernambuco-ZAPE, desenvolvido pela Secretaria de Produção Rural e Reforma Agrária de Pernambuco em convênio com a Embrapa Solos Unidade de Execução e Pesquisa (UEP) Recife, onde o mesmo apresenta condições agroecológicas distintas, com variações em termos de clima, vegetação, solo, recursos hídricos, etc. Para fins de realização do trabalhou considerou apenas dados de solos com potenciais aptos para a exploração agrícola em todo o estado de Pernambuco.

A espécie utilizada neste estudo foi $A$. numulária L., que possui origem na Austrália, conforme a caracterização realizada por Franclet \& Le Houérou (1971), onde existem mais de 400 espécies. É uma espécie que se desenvolve em locais que apresentam precipitação entre 100 a $500 \mathrm{~mm} \cdot \mathrm{ano}^{-1}$, temperatura variando de 25 a $40^{\circ} \mathrm{C}$ e altitude de 0 a $1500 \mathrm{~m}$, em quase todos os tipos de solo (Falasca et al., 2013). Com base nessas informações, assumiram-se dados hídrico- térmicos e de solo para a definição do potencial agrícola no estado de Pernambuco à exploração da A. numularia. Para isso foram adotadas as classes de aptidão da cultura para esta espécie descritas na Tabela 1 .

Tabela 1. Classes de aptidão referentes às exigências da cultura da Atriplex numularia L.

\begin{tabular}{c|c|c}
\hline $\begin{array}{c}\text { Precipitação } \\
\text { pluviométrica } \\
(\mathbf{m m})\end{array}$ & $\begin{array}{c}\text { Temperatura } \\
\left({ }^{\mathbf{C}} \mathbf{C}\right)\end{array}$ & $\begin{array}{c}\text { Pedoclimático } \\
(\text { Solo })\end{array}$ \\
\hline$<400$ & $<24$ & Restrita \\
$400-650$ & $>24$ & Regular \\
$650-1200$ & & $\begin{array}{c}\text { Marginal } \\
<1200\end{array}$ \\
& & Solos inaptos \\
\hline
\end{tabular}

A estimativa da produtividade foi realizada utilizando indicadores de eficiência do uso da água, com base na precipitação pluviométrica, como também técnicas de manejo como fatores de idade e densidade das plantas. A eficiência no uso da água é a relação entre a produção de biomassa, em kg.ha ${ }^{-1} \cdot \mathrm{mm}^{-1}$ (Figura 2A), a idade foi considerada a partir do momento do primeiro corte em relação aos meses seguintes (Figura 2B), e a densidade como a razão do número de plantas por hectare (Figura 2D).

A estimativa dos parâmetros de produtividade da $A$. nummularia basearam-se em estudos de vários autores (Barroso et al., 2006; Glenn et al., 1998; Porto et al., 2004; Silva, 2010), de tal modo que foi ajustado para cada fator um modelo matemático, onde, para a densidade, adotaram-se valores de 625, 1111, 2500 e 10000 plantas.hectare $^{-1}$; para avaliar a produtividade da 
cultura foi considerado um valor inicial padrão de 625 plantas hectares $^{-1}$. Para o fator corte fez-se a relação entre o intervalo de corte, em meses, e a produtividade em kg.ha- ${ }^{-1}$ da cultura, sendo que, tanto para o fator densidade como idade de plantas, foi expressa em proporção, uma variável em relação à outra.

Para a eficiência do uso da água foi utilizado modelos matemáticos, porém fez-se a sua relação com a produtividade da cultura, considerando que, a cada milímetro de água absorvido pela planta, a mesma irá converter em biomassa. No modelo matemático assumiu-se a precipitação efetiva, com perdas de $25 \%$ em decorrência de escoamento, drenagem profunda, evaporação, entre outros fatores (Araya et al., 2011). Ajustou-se, ainda, uma equação para a condutividade elétrica, relacionando os seus valores com a produtividade da cultura, em kg.ha- ${ }^{-1}$ (Figura 2C).

Finalmente, obteve-se o modelo matemático de Produtividade Estimada Ajustada
(PEA) pela Equação 1, onde foi considerada a eficiência do uso da água, idade de corte, precipitação efetiva e condutividade elétrica. Ressalta-se que, essa última, foi incorporada ao modelo, porém, não aplicada na estimativa regional da produtividade de Pernambuco, devido à ausência de dados de condutividade elétrica do Estado. Contudo, pode ser aplicado a regiões que possuam essa informação disponível.

Após a obtenção dos dados de chuva, temperatura e da equação de PEA, os mapas foram espacializados utilizando o sistema de informações geográficas-SIG, ArcGIS versão 9.3. Para a manipulação dos dados foi importado o banco de dados (planilha Excel) e convertido em shapefile (SHP), que é a extensão compatível com o software. Posteriormente, foi realizada a interpolação dos dados para obtenção dos mapas de chuva, temperatura, zoneamento climático, pedológico, pedoclimático e de produtividade da cultura.
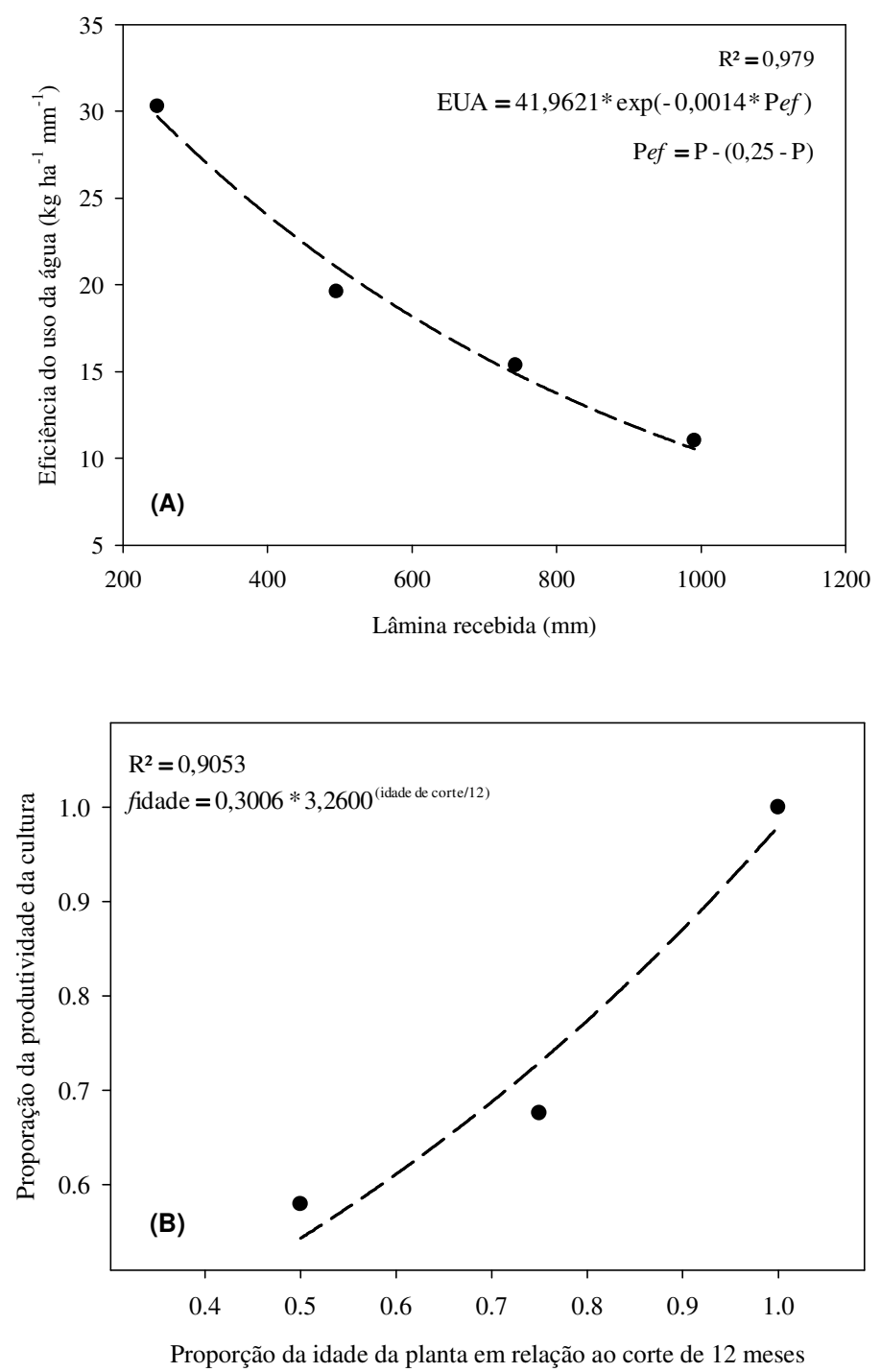

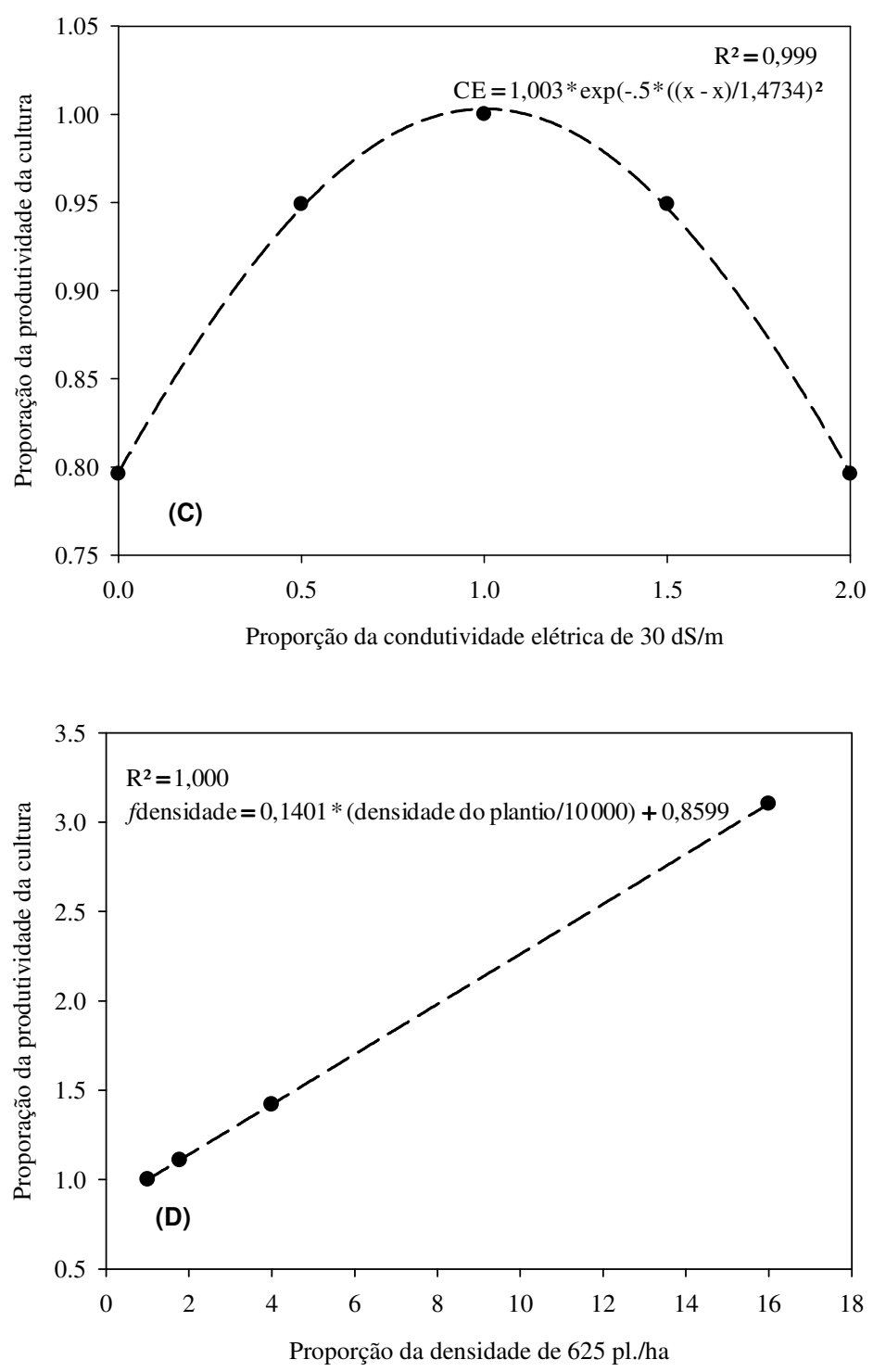

Figura 2. Correlação entre os valores de: A. Eficiência do uso da água e lâmina recebida; B. Produtividade da cultura em relação à condutividade elétrica; C. Produtividade da cultura em relação ao intervalo de corte; D. e Produtividade da cultura em relação a densidade de plantio para o estado de Pernambuco.

PEA $=\left(\right.$ EUA.P $\left.{ }_{\text {ef }}\right) \cdot\left(f_{\text {idade }}\right) \cdot\left(f_{\text {densidade }}\right) \cdot\left(f_{\text {CE }}\right) \quad$ Eq. $(1)$

onde PEA = é a produtividade estimada ajustada $(\mathrm{kg} \mathrm{MS} / \mathrm{ha}) ; \mathrm{EUA}=$ eficiência do uso da água $\left(\mathrm{kg} / \mathrm{m}^{3}\right) ; \mathrm{P}_{\mathrm{ef}}=$ Precipitação efetiva $(\mathrm{mm}) ; f_{\text {idade }}=$ fator de ajuste da produtividade à idade de corte; $f_{\text {densidade }}=$ fator de ajuste da produtividade à densidade de plantio; $f_{\mathrm{CE}}=$ fator de ajuste da produtividade à densidade de plantio.

\section{Resultados}

Considerando os dados de chuva, temperatura do ar e a equação de produtividade estimada ajustada-PEA, os mapas de temperatura do ar e a precipitação foram espacializados (Figura 3A), mostrando duas classes térmicas, uma com temperaturas superiores a $24^{\circ} \mathrm{C}$ e outra com valores abaixo de $24^{\circ} \mathrm{C}$. Os valores superiores a $24^{\circ} \mathrm{C}$, uma condição ideal para o crescimento e desenvolvimento da cultura, encontram-se na mesorregião litorânea do estado e parte do sertão, na região do São Francisco. Por outro lado, as menores temperaturas são observadas na mesorregião agreste e parte do sertão pernambucano.

A precipitação foi classificada em quatro classes (Figura 3B), onde a mínima foi inferior a $400 \mathrm{~mm}^{\mathrm{anono}}{ }^{-1}$, não identificada em nenhuma mesorregião do estado. As maiores magnitudes são evidenciadas na mesorregião litorânea, que compreende a zona da mata e a parte metropolitana do Recife. Para as outras regiões do estado, há uma variação entre $400 \mathrm{~mm}$ e $1200 \mathrm{~mm}$ (Figura 3B). Essa variabilidade espacial, e temporal, tem sido afirmada por Rodrigues et al. 
(2014), em estudo realizado para o estado de Pernambuco.

De posse das duas bases de dados, da temperatura do ar e precipitação, foi possível realizar a intersecção dos dois mapas, proporcionando a criação do mapa climatológico (Figura 4A). Neste mapa, identificam-se as áreas adequadas, marginais, com restrição hídrica e térmica e ótima. Constatou-se que praticamente toda mesorregião do sertão do São Francisco e pequena área que compreende o agreste e zona mata é restrita por condições hídrica e térmica. Para as outras classes, como adequada e ótima, englobou o agreste e sertão pernambucano. Enquanto, na zona da mata e metropolitana do Recife, apresenta-se como área marginal.
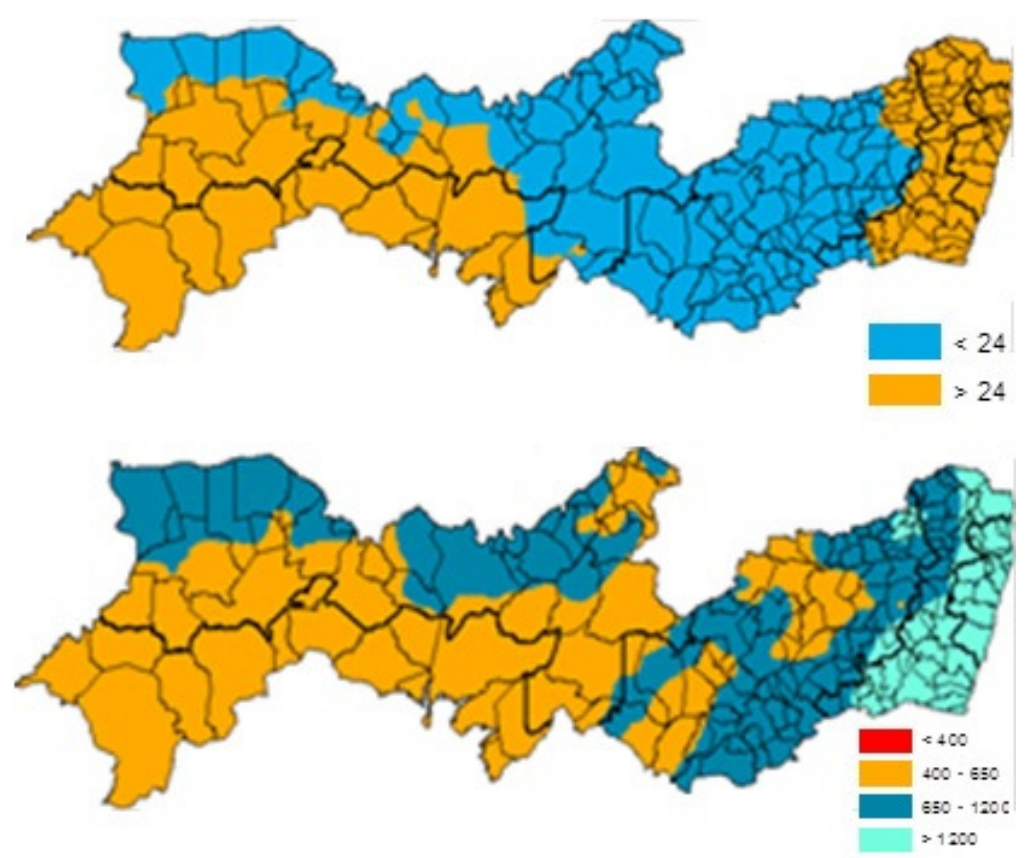

Figura 3. Espacialização do mapa de temperatura média do ar (A) e precipitação pluviométrica (B) para o estado de Pernambuco, em 2017. Fonte: elaborado pelo o autor. (2017).
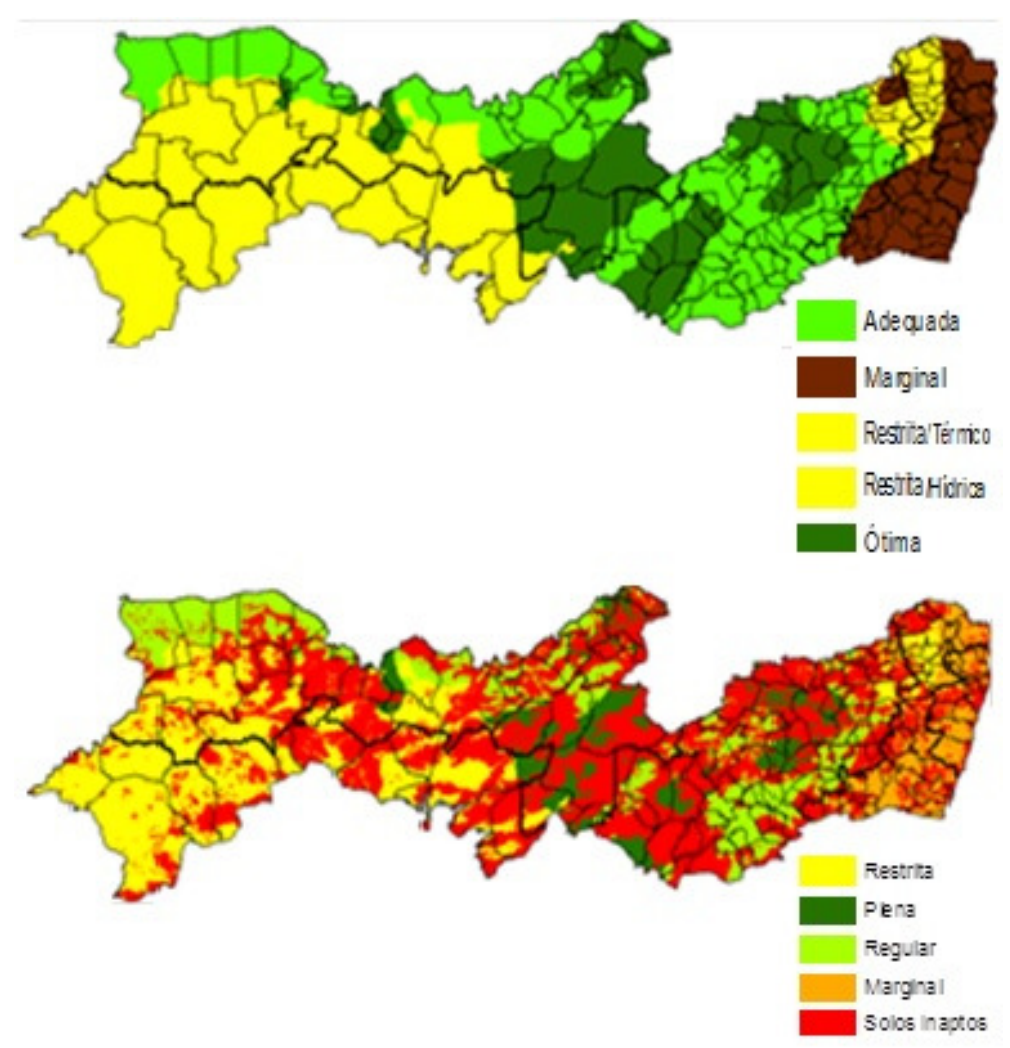

Figura 4. Espacialização do mapa climatológico (A) e pedoclimático (B) para o estado de Pernambuco, em 2017. Fonte: elaborado pelo o autor. (2017). 
Em relação às condições de solo e clima, o mapa pedoclimático (Figura 4B) mostra a classificação do estado de Pernambuco em zonas: restrita, plena, regular, marginal e inapta. Fica evidente o predomínio dessa última, em todas as mesorregiões do estado, devido às limitações relativas aos tipos de solo. Nas áreas restritas predominaram o sertão e o São Francisco, em relação às áreas marginais, às duas regiões litorâneas, à zona da mata e à região metropolitana. Por sua vez, as classes: plena e ótima estão inseridas quase que exclusivamente no agreste e sertão pernambucano.

Considerando os parâmetros de Eficiência do Uso da Água-EUA, densidade, idade de corte, e condutividade elétrica da água, os quais foram utilizados na Equação 1 da PEA, foram espacializados os mapas de produtividade da $A$. nummularia no estado de Pernambuco.

A variabilidade da produção espacial da erva sal, para o estado de Pernambuco em diferentes idades de corte e densidade de 625, 1111,2500 e 10000 plantas por hectare, pode ser observada na Figura 5A-D (12 meses); E-H (9 meses) e I-L (6 meses).

No corte da cultura com 12 meses percebe-se que, a produtividade varia entre $5500 \mathrm{e}$ $10500 \mathrm{~kg} \cdot \mathrm{ha}^{-1}$. Quanto mais aumenta a densidade das plantas neste intervalo de tempo, ocorre aumento na produtividade. As áreas com produtividade abaixo de $5500 \mathrm{~kg} \cdot \mathrm{ha}^{-1}$ quase não aparecem no mapa. Parte das regiões do Sertão e São Francisco são adequada a implantação da cultura, assim como a meso região do agreste, que atinge uma produção superior a $10500 \mathrm{~kg} \cdot \mathrm{ha}^{-1}$.

Diminuindo o tempo de corte para 9 meses e mantendo as densidades (Figura 5E-H) pode-se observar que continuam aumentando as áreas propícias ao cultivo da erva sal. Embora tenha sido constatada uma redução na produtividade da cultura em relação ao tempo de corte aos 12 meses. O mesmo acontece quando se reduz ainda mais o tempo de corte, para 6 meses (Figura 5I-L).

\section{Discussão}

A maior parte das regiões do estado se mostra ideal para o cultivo da erva-sal, com exceção da região metropolitana, por possuir regime de precipitação elevado, > $1200 \mathrm{~mm}$, apresentando-se como área marginal ao cultivo, de acordo com o mapa climatológico, sendo 500 mm o ideal.
Resultado semelhante foi obtido por Moura et al. (2011) para a palma forrageira no estado de Pernambuco, onde as mesorregiões que compreendem a zona da mata e metropolitana do Recife se mostraram inadequadas para o cultivo. Assim, possivelmente como a palma forrageira, a A. nummularia é uma espécie adaptada ás regiões com precipitações mais baixas; aquelas com precipitação acima de $1200 \mathrm{~mm} \cdot \mathrm{ano}^{-1}$ não são recomendadas para o seu cultivo.

Em estudos desenvolvidos no Vale do São Francisco, Silva (2010) verificou que, plantas de A. nummularia com densidade de plantio de 10000 plantas por hectares e intervalode corte de 6 meses, apresentam melhor produtividade em um menor intervaldo de tempo, em relação a densidades menores e cortes com maior intervalo de tempo. Resultado semelhante foi observado neste trabalho, de fato a produtividade superior a $5300 \mathrm{~kg} \cdot \mathrm{ha}^{-1}$ é maior nas condições de intervalo de corte de 6 meses e densidade de 10000 plantas por hectares, com uma maior área de abragência em relação a outras faixas de produtividades.

De acordo com Baghestani Meybodi et al. (2006), avaliando efeitos de diferentes espaçamentos, altura de corte e intervalos de cortes, (tres vezes ao ano) durante dez anos, observaram que à medida que aumenta a intensidade de corte, o vigor das plantas diminui. Constata-se que o tempo de corte é uma variável importantíssima para que haja maior acúmulo de biomassa na planta, consequentemente uma maior produtividade. Porém, Mahdavi et al. (2009) relatam que maiores produções de forragem foram encontradas quando os cultivos foram mais adensados.

Silva (2010) afirma que, além do tempo de corte, a densidade é uma variável que interfere na produtividade, visto que, à medida que se diminui a quantidade de plantas por área, observase um aumento da produtividade. Vale ressaltar, também, que essas variações são influenciadas pelo manejo, condução de práticas culturais e espaçamento das plantas.

Embora áreas com potencial pleno, onde a cultura expressa seu potencial máximo de produtividade, a produtividade é mais reduzida, devido às limitações térmicas ou hídricas, salientando que podem ser adotadas técnicas de irrigação onde o fator restritivo for o hídrico. 


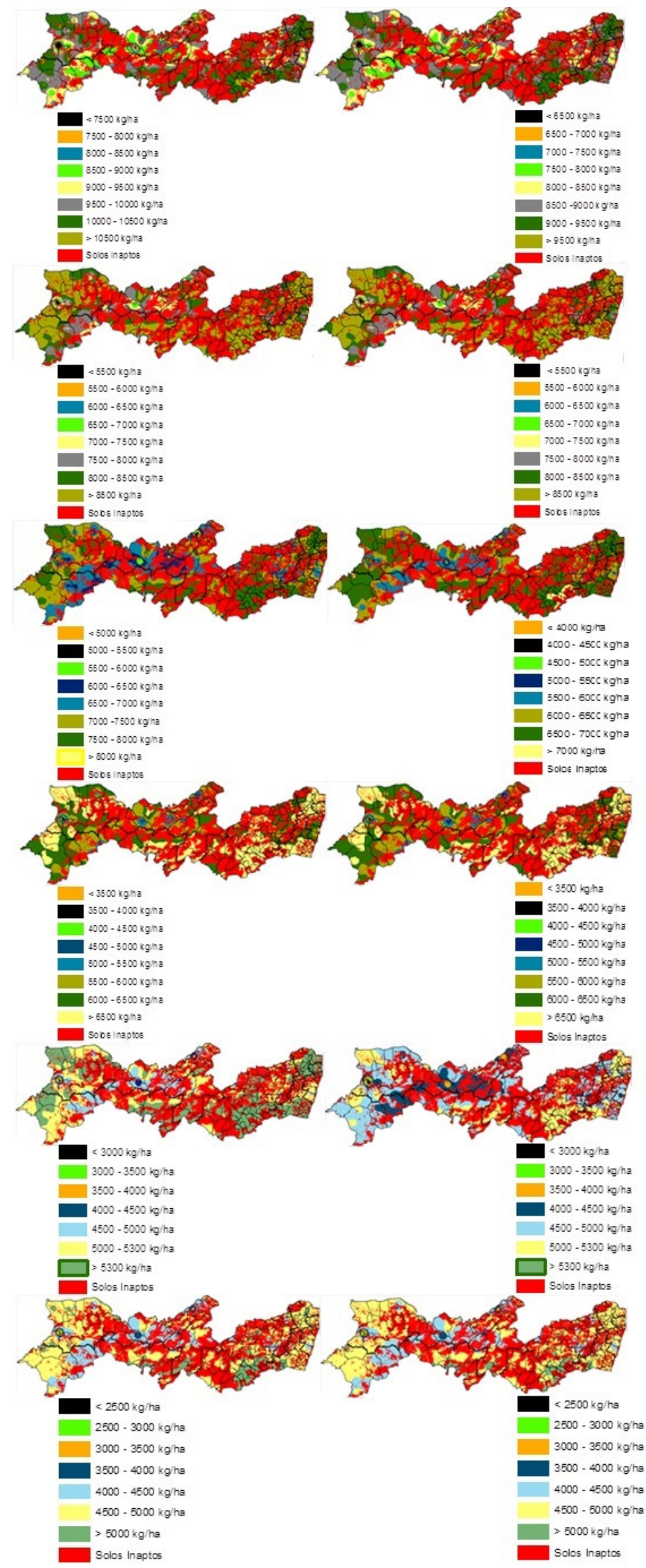

Figura 5. Espacialização da produtividade estimada ajustada, em função da idade de corte e densidade de plantio para a cultura da erva sal no estado de Pernambuco, em 2017. Fonte: elaborado pelo o autor. (2017). 


\section{Conclusão}

O estado de Pernambuco mostra grandes extensões de área com aptidão para o cultivo da erva sal, expressando maior potencial em parte do Sertão e Vale do São Francisco, apesar da variação na produtividade nas diferentes regiões do Estado.

\section{Agradecimentos}

À Universidade Federal Rural de Pernambuco-UFRPE, Unidade Acadêmica de Serra Talhada-UAST no uso de suas instalações para o desenvolvimento da pesquisa.

\section{Referências}

AGANGA, A. A.; MTHETHO, J. K.; TSHWENYANE, S. 2003. Atriplex nummularia (Old Man Saltbrush): A potential forage crop for arid regions of Botswana. Paquistão, Jornal de Nutrição. v. 2, p. 72-75.

ALVARES, C. A.; STAPE, J. L.; SENTELHAS, P. C.; GONÇALVES, J. L. DE M. 2012. Modeling monthly mean air temperature for Brazil. Springer.

BAGHESTANI MEYBODI, M.; ABD ELAHI, J.; MIRJALILI, M. R. T. 2006. The effects of plant row spaces and cutting methods on vigority of Atriplex lentiformis in Yazd province. Desert, v. 11, n. 1, p. 157-166.

BARROSO, D. D.; ARAÚJO, G. G. L. DE; SILVA, D. S. DA; MEDINA, F. T. 2006. Resíduo desidratado de vitivinícolas associado a diferentes fontes energéticas na alimentação de ovinos: consumo e digestibilidade aparente. Revista Ciência e Agrotecnologia, v. 30, n. 4, p. 767-773.

\section{FALASCA S. L.; PIZARRO, M. J.; MEZHER,} R. N. 2013. The agro-ecological suitability of Atriplex nummularia and A. halimus for biomass production in Argentine saline dry lands. Int. J. Biometeorol., v. 58, n. 7, p. 1433-1441.

FRANCLET, A.; LE HOUÉROU, H. N. Les Atriplex en Tunisieet en Afrique du Nord. Roma: FAO, 1971. (FAO Report Technique, 7), 249p.

GLENN, E.; TANNER, R.; MIYAMOTO, S.; FITZSIMMONS, K.; BOYER, J. 1998. Water use, productivity and forage quality of the halophyte Atriplex nummularia grown on saline waste water in desert environment. Journal of Arid Environments, v. 38, n. 9, p. 45-62.
LEAL, I. G.; ACCIOLY, A. M. DE A.; NASCIMENTO, C. W. DO; FREIRE, M. B. G. DOS S.; MONTENEGRO, A. A. DE A.; FERREIRA, F. DE L. 2008. Fitorremediação de solo salino sódico por Atriplex nummularia e gesso de jazida. R. Bras. Cien. Solo, v. 32, p. 1065-1072.

MAHDAVI, $\quad$ K.; $\quad$ SANADGOL, A.; AZARNIVAND, H.; KAFAKI, S. B.; JAFARI, M.; MALEKI, M.; MALEKIAN, A. 2009. Effects of removing aerial biomass 45 and density on carbon sequestration and weight of Atriplex lentiformis. Asian Journal of Plant Sciences, v. 8, n. 2, p. 183-186.

MAIA, S. M.; SILVA, V. A.; LOGES, V.; SILVA, V. M. B.; MAIA, L. M. C.; CASTRO, A. C. R. 2003. Potencial de uso de espécies de Atriplex no paisagismo. In: Congresso Brasileiro de Floricultura e Plantas Ornamentais, 14. Anais. Lavras, p. 15.

MOURA, M. S. B. SOUZA, L. S. B. DE; SILVA, T.G. F. DA; SÁ, I. I. S. 2011. Zoneamento agroclimático da palma forrageira para o Estado de Pernambuco. Petrolina: Embrapa Semiárido.

PORTO, E. R.; AMORIM, M. C. C. DE; ARAÚJO, O. J. 2000. Potencialidades da erva-sal (Atriplex nummularia) irrigada com o rejeito da dessalinização de água salobra no semi-árido brasileiro como alternativa de reutilização. In: XXVII Congresso Interamericano de Engenharia Sanitária e Ambiental, Rio de Janeiro.

PORTO, E. R.; AMORIM, M. C. C. DE; SILVA JÚNIOR, L. G. DE A. 2001. Uso do rejeito da dessalinização de água salobra para irrigação da erva-sal (Atriplex nummularia), Revista Brasileira Engenharia Agrícola Ambiental p. 111-114.

RODRIGUES, C. T. A. 2014. Variabilidade Espacial e Temporal da Chuva no Estado Estado de Pernambuco. In: $9^{\circ}$ Simposio Brasileiro de Capitação e Manejo de Água de Chuva.

SILVA, J. R. R. 2010. Produção e características estruturais da erva-sal, submetida a diferentes densidades de plantio e intervalos de cortes. Petrolina-PE, Dissertação UNIVASF.

SILVA, V.A.; MAIA, S.M.; LOGES, V.; MAIA, L.M.C.; SILVA, V.M.B.; COSTA, A.S. \& CRUZ, S.R.C.L. 2003. Potencial de uso de espécies de Atriplex na floricultura. In.: Congresso Brasileiro 
Journal of Environmental Analysis and Progress V. 02 N. 03 (2017) 302-311

de Floricultura e Plantas Ornamentais, Lavras, 2003. Anais... Lavras, pp. 48-48.

SOUZA, E. R. 2010. Fitorremediação de neossolo flúvico sódico salino de Pernambuco com Atriplex nummularia. Tese (Doutorado em Ciência do Solo), Universidade Federal Rural de Pernambuco. 